\title{
Transparence de la qualité pour les médecins et les patients
}

\section{Le contexte}

De nombreuses activités en matière de qualité sont menées à bien dans les hôpitaux, les cabinets médicaux et les réseaux de médecins. Elles sont souvent trop peu visibles pour les patients et le public intéressé. La FMH voit donc là une nécessité d'agir et se mobilise en faveur d'une plus grande transparence de la qualité des prestations médicales. Les médecins accomplissent une part importante du travail relatif à la qualité médicale. C'est pourquoi ils ont leur mot à dire lorsqu'il s'agit de déterminer quelles données doivent être collectées, évaluées et publiées. La FMH occupe une fonction coordinatrice au sein du corps médical. A ce titre, elle entretient des échanges avec les sociétés de discipline médicale qui définissent les activités relatives à la qualité dans leurs domaines spécifiques et les indicateurs destinés à la mesurer. La FMH représente le corps médical et élabore, en collaboration avec ses partenaires tarifaires, les fondements d'une transparence de la qualité bien comprise.

\section{La position de la FMH}

La transparence de la qualité est utile à la gestion interne de la qualité

La transparence de la qualité est le fondement de la gestion interne de la qualité. Un travail systématique et continu sur la qualité aide celle ou celui qui le pratique à déceler ses forces et ses faiblesses. Des prestations médicales efficaces, adéquates et économiques impliquent une évaluation bien pensée de la qualité, qui permette de se prononcer sur la qualité des traitements. L'objectif prioritaire doit être l'amélioration des résultats et non la diminution des coûts. L'un des bénéfices importants du travail qualitatif est la sécurité des patients. Des processus visant à limiter les erreurs préservent les patients de dommages et contribuent simultanément au caractère économique des prestations. Une culture transparente de la qualité profite à tous: patients, fournisseurs de prestations et assureurs.

Correspondance: Secrétariat général de la FMH Département DDQ Elfenstr. 8 CH-3000 Berne 15

ddq@fmh.ch

\section{Concurrence fondée sur la qualité et pas uniquement sur les prix}

L'évaluation et la publication d'indicateurs de qualité permettent de comparer les fournisseurs de prestations. Cela devrait permettre au patient de choisir plus facilement le médecin ou l'institution qui lui convient. La FMH s'engage en faveur d'une concurrence non biaisée: les données relatives à la qualité doivent être collectées et évaluées de manière correcte. Il convient de ne pas négliger les aspects du traitement médical qui ne figurent pas au centre de l'évaluation qualitative, et les activités dans le domaine de la qualité ne doivent pas poursuivre uniquement des objectifs à court terme. Il faut continuer à promouvoir les innovations. La FMH tient à aménager, en collaboration avec le législateur et ses partenaires tarifaires, les conditions qui rendront possible une concurrence loyale fondée sur la qualité des prestations médicales.

\section{Transparence de la qualité pour les patients}

La publication de données qualitatives ne doit pas avoir pour but de satisfaire aux exigences des médias. Il s'agit bien plus de tenir compte des besoins des patientes et des patients. La FMH encourage une transparence adaptée au groupe-cible: toutes les données qualitatives ne revêtent pas la même utilité et ne sont pas sujettes à la même interprétation pour différents interlocuteurs. Dès lors, toutes les données concernant la qualité ne se prêtent pas à une publication large. Des études de la FMH permettent de mieux connaître les besoins d'information des patients quant à la qualité des traitements médicaux.

La qualité est aussi importante lors de la collecte, de l'évaluation et de la publication des données Des données sur la qualité inexactes ou mal analysées empêchent le corps médical de démontrer la bonne qualité de ses prestations. Elles n'aboutissent pas à l'amélioration souhaitée et rendent impossible une saine concurrence entre fournisseurs de prestations. Enfin, les patients sont induits en erreur et leur confiance dans le système de santé s'en trouve amoindrie. Seule la publication de données de qualité exactes et intelligibles pour leurs destinataires déploie des effets positifs. Les chiffres n'ont de force probante que s'ils sont accompagnés des outils d'interprétation adéquats. C'est pourquoi la publication de données sur la qualité doit être régie par les critères élaborés à cette fin par un groupe de travail, placé sous l'égide de l'Académie suisse des sciences médicales. La publication de telles données n'a de sens que si elles représentent réellement la qualité des traitements. Pour que les indicateurs puissent délivrer des indications sur la qualité de l'activité médicale, ils doivent donc impérativement être définis en collaboration avec le corps médical. 